\title{
Performance Optimal and Robust Design of an Idle-Speed Controller Considering Physical Uncertainties
}

\author{
Eduard Popp, Mathias Tantau, Mark Wielitzka, and Tobias Ortmaier \\ Institute of Mechatronic Systems \\ Leibniz University of Hanover \\ An der Universität 1, 30823 Garbsen, Germany \\ \{eduard.popp, mathias.tantau, mark.wielitzka, tobias.ortmaier\} \\ @imes.uni-hannover.de
}

\author{
Dennis Giebert \\ IAV Automotive Engineering \\ Rockwellstraße 5, 38518 Gifhorn, Germany \\ giebert@iav.de
}

\begin{abstract}
Modern passenger vehicles are equipped with a great number of control functions targeting versatile performance aspects like safe drive-ability, comfortable or sporty ride concerning assistance systems or a proper adjustment of engine control functions in order to prevent noise vibration and harshness issues. In this paper a methodology for a performance optimal and robust controller design is presented. This methodology is applied on a given idle-speed controller implementation using a detailed nonlinear drive train model in closed loop considering physical parameter uncertainties. The results are discussed with exemplary selected performance measures.
\end{abstract}

Index Terms - Automotive application, robust control design, PI-controller, sensitivity analysis, parameter space approach.

\section{INTRODUCTION}

Modern passenger vehicles are equipped with a great number of control functions targeting versatile aspects like a safe driveability, comfortable or sporty ride concerning assistance systems following [1] or a good adjustment of engine control functions in order to prevent noise vibration and harshness issues as discussed by [2] regarding the idle speed controller. This paper is focused on the latter target. One way to prevent $\mathrm{NVH}$ phenomena is to design a novel controller structure or reduce the delay time as discussed in e.g. in [2]. In contrast to that, the goal of this paper is to find a robust parameterization of a given idle-speed controller implementation. For this reason certain criteria are required that evaluate the closed loop behaviour in order to distinguish between an acceptable and a rejectable parametrization. In order to ensure better understanding and acceptance for users of this approach, the performance requirements are calculated in time domain with respect to measured signals. The presented approach is performed on the basis of a detailed nonlinear model of the closed loop system and takes into account physical uncertainties like the quality of the engine calibration, tolerances of the drive train components caused by ageing and manufacturing and different loads states of the vehicle. In terms of interactions with other control functions like the fuel balance controller or the anti-jerk control, these and further functions may be integrated into the given software structure. Robust design of an vehicle idle speed controller has been investigated in numerous papers. At this point of view two distinct systems are regarded: on one hand engine dynamics are focused considering throttle control and on the other hand drive train dynamics are focused with respect to accurate modelling of the clutch dynamics and backlash due to the gearbox and further transmission components. Regarding these approaches, first the quantitative feedback control by [3] considering an intake air path as simple linear second order system in closed loop with two uncertain parameters must be mentioned. The controller design takes place in frequency domain by means of the nichols chart and the subsequently analysis in time domain. In addition the $H_{\infty}$ approach and $\mu$-synthesis have received much attention considering unstructured and structured uncertainties [4], [5]. It must be noticed that stability and performance demands are analysed in frequency domain by means of the sensitivity and complementary sensitivity transfer function such that physical performance measures cannot be taken into account directly, which have major significance in automotive application. Moreover, stability tests in frequency domain provide conservative results with respect to maximum permissible uncertainty intervals [6]. The main draw back is that for the design linearized models are required. In case of explicitly incorporating nonlinearities like backlash, coulomb friction, nonlinear crankshaft dynamics or nonlinearities within the controller structure there are no alternatives to simulations in time domain. Under this assumption performance portraits are employed, where control parameters within a certain region are grid and evaluated concerning particular performance measures [7], [8]. In terms of further elaboration this approach is taken in order to meet the initially stated requirements for an automated controller design in an automotive application.

This paper is organized as follows. In section II the utilized nonlinear vehicle model is introduced. In section III the performance and robustness measures are defined and the proposed methodology is described. The results are presented 
in section IV and finally a conclusion is given in section $\mathrm{V}$.

\section{VEHICLE MODEL}

In this paper especially an accurate approximation of the dynamic behavior at idle-speed is decisive. For this purpose the drive train is assumed as three mass system that is shown in Fig. 1 and is based on [2] and [9]. $J_{\mathrm{p}}$ represents the mass of the crankshaft and the primary part of the dual mass flywheel (DMF) and $J_{\mathrm{s}}$ consists of the secondary side of the DMF and a part of the rotational mass of the gear box. The DMF furthermore exhibits coulomb friction characteristic, which is represented by $T_{\text {clmb }}$. The spring is commonly an multistage arc spring with backlash of width $\varepsilon_{\text {clu }}$. As the engine torque remains at idle speed with low variations using a linear stiffness $c_{\text {clu }}$ and damping coefficient $d_{\text {clu }}$ is sufficient. The clutch is considered to be engaged. The output shaft of the gearbox is connected with the wheels $J_{\mathrm{w}}$ respectively the vehicle mass by an elastic drive shaft corresponding to the stiffness $c_{\mathrm{drv}}$ and damping $d_{\mathrm{drv}}$. Due to the gearbox a further backlash of width $\varepsilon_{\mathbf{d r v}}$ must be taken into account. Torque losses $T_{\text {loss }}$ are assumed to be constant at idle-speed. This leads to the equation of motion:

$$
\begin{aligned}
J_{\mathrm{p}} \ddot{\varphi}_{\mathrm{p}} & =T_{\mathrm{eng}}-T_{\mathrm{loss}}-T_{\mathrm{clu}}-T_{\mathrm{d}_{\mathrm{clu}}}-T_{\mathrm{clmb}} \\
J_{\mathrm{s}} \ddot{\varphi}_{\mathrm{s}} & =T_{\mathrm{c}_{\mathrm{clu}}}+T_{\mathrm{d}_{\mathrm{clu}}}+T_{\mathrm{clmb}_{\mathrm{clu}}}-\frac{1}{i_{\mathrm{g}}}\left(T_{\mathrm{c}_{\mathrm{drv}}}+T_{\mathrm{d}_{\mathrm{drv}}}\right) \\
J_{\mathrm{w}} \ddot{\varphi}_{\mathrm{w}} & =T_{\mathrm{c}_{\mathrm{drv}}}+T_{\mathrm{d}_{\mathrm{drv}}}
\end{aligned}
$$

with the input torque $T_{\text {eng }}$ and load torque $T_{\text {load }}$. With respect to a physical backlash model by [10] two further states need to be introduced:

$$
\lambda_{\text {clu }}= \begin{cases}\varepsilon_{\text {clu }} \operatorname{sign}\left(\tau_{\text {clu }}\right), & \left|\tau_{\text {clu }}\right| \geq \varepsilon_{\text {clu }} \\ \tau_{\text {clu }}, & \left|\tau_{\text {clu }}\right|<\varepsilon_{\text {clu }}\end{cases}
$$

with $\tau_{\text {clu }}=\varphi_{\mathrm{p}}-\varphi_{\mathrm{s}}$ and

$$
\dot{\lambda}_{\text {clu }}= \begin{cases}\min \left(0, \dot{\delta}_{\text {clu }}\right), & \lambda_{\text {clu }}=-\varepsilon_{\text {clu }} \\ \dot{\delta}_{\text {clu }}, & \left|\tau_{\text {clu }}\right|<\varepsilon_{\text {clu }} \\ \max \left(0, \dot{\delta}_{\text {clu }}\right), & \lambda_{\text {clu }}=\varepsilon_{\text {clu }}\end{cases}
$$

with $\dot{\delta}_{\text {clu }}=\dot{\tau}_{\text {clu }}-\frac{c_{\text {clu }}}{d_{\text {clu }}}\left(\tau_{\text {clu }}-\lambda_{\text {clu }}\right)$. In equivalence to the coupling of the DMF the states for the backlash of the drive shaft $\lambda_{\mathrm{drv}}$ and $\dot{\lambda}_{\mathrm{drv}}$ are calculated in a similar way

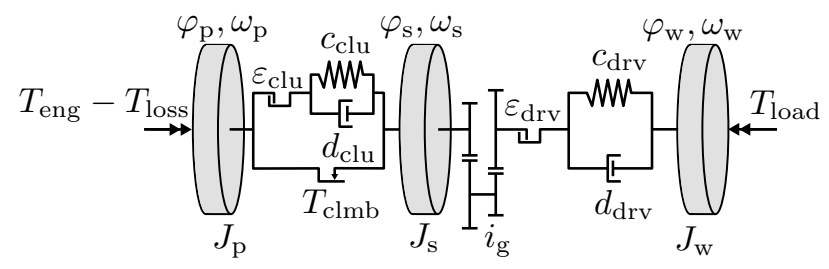

Fig. 1. Model structure of the drivetrain. considering $\tau_{\mathrm{drv}}=\frac{\varphi_{\mathrm{s}}}{i_{\mathrm{g}}}-\varphi_{\mathrm{w}}$ and $\dot{\delta}_{\mathrm{drv}}=\dot{\tau}-\frac{c_{\mathrm{drv}}}{d_{\mathrm{drv}}}\left(\tau_{\mathrm{drv}}-\lambda_{\mathrm{drv}}\right)$. Finally the torques from (1)-(3) can be obtained as follows:

$$
\begin{aligned}
T_{\text {clu }}= & c_{\text {clu }}\left(\tau_{\text {clu }}-\lambda_{\text {clu }}\right)+d_{\text {clu }}\left(\dot{\tau}_{\text {clu }}-\dot{\lambda}_{\text {clu }}\right) \\
& +T_{\text {clmb }} \operatorname{sign}\left(\dot{\tau}_{\text {clu }}\right) \\
T_{\text {drv }}= & c_{\text {drv }}\left(\tau_{\text {drv }}-\lambda_{\text {drv }}\right)+d_{\text {drv }}\left(\dot{\tau}_{\text {drv }}-\dot{\lambda}_{\text {drv }}\right) .
\end{aligned}
$$

The engine torque $T_{\text {eng }}$ consists of the combustion torque $T_{\mathrm{cmb}}$, the compression and expansion torque $T_{\mathrm{c} / \mathrm{e}}$ and the mass torque $T_{\mathrm{j}}$. As they arise from non-continuous combustion and non-linear torque transmission on the crankshaft drive, they depend on the angular position $\varphi_{\mathrm{p}}$ and speed of the crankshaft $\omega_{\mathrm{p}}$ as follows:

$$
T_{\mathrm{eng}}=\sum_{i=1}^{N_{\mathrm{cyl}}} T_{\mathrm{cmb}}\left(\varphi_{\mathrm{p}, i}\right)+T_{\mathrm{c} / \mathrm{e}}\left(\varphi_{\mathrm{p}, i}\right)+T_{\mathrm{j}}\left(\varphi_{\mathrm{p}, i}, \omega_{\mathrm{p}, i}\right),
$$

with $T_{\mathrm{cmb}}=T_{\mathrm{ecu}} K_{\mathrm{eng}}$. According to the accuracy of the engine calibration at certain operation points the desired torque of the controller output $T_{\text {ecu }}$ is applied with respect to the gain $K_{\text {eng }}$ on the drive train. This parameter is introduced for robustness reasons. For more details in the terms of (8) please see [11].

\section{Controller Design}

For the introduced controller design approach in this paper the entire closed loop system is required. This contains the given implementation of the idle speed controller on the device, a nonlinear model of the combustion engine and the vehicle drive train dynamics. In modern vehicles the idle speed controller is a PI-controller, that contains nonlinear parts like engine speed based gain scheduling and saturation functions. Designing a controller typically has two aims. First, the control parameters shall be adjusted optimal concerning certain performance measures, which will be focused in the first subsection. Second, a good robustness against model uncertainties must be ensured that will be addressed subsequently by introducing physical parameter uncertainties. In this paper we will take both aims in a closer view and compare the results with each other.

\section{A. Performance Measures}

In terms of an optimal controller design commonly the aim is to achieve good disturbance rejection, noise annotation, reference tracking and low energy consumption of the actuator, which are comprehended as performance requirements. In order to meet all of them, a multicriterial optimization is required. In this paper we assume to satisfy a maximum settling time with a tolerance band and a maximum overshoot of certain processed signals that are obtained on the basis the measured engine speed $n_{\mathrm{p}}$ and the measured wheel speed $n_{\mathrm{s}}$ as shown in Fig. 2 by means of the presented simulation model. In case of the wheel speed the delay time due to filters and the gear ratio with respect to the engine speed 
is corrected. Regarding Fig. 2(b) two processed signals are pictured. The upper signal is calculated as $n_{1}=n_{\mathrm{w}}-w$, with the reference value $w$. The lower signal is $n_{2}=n_{\mathrm{p}}-n_{\mathrm{w}}$. On this basis the following performance measures are defined:

1) settling time $t_{\varepsilon_{1}}$ of $n_{1}$ into a band width $\varepsilon_{1}$,

2) overshoot $h$ of $n_{1}$,

3) settling time $t_{\varepsilon_{2}}$ of $n_{2}$ into a band width $\varepsilon_{2}$.

The signals have to settle in within the boundaries that are drawn in solid black lines. The measured performance values correspond with the solid red lines in case of the settling time and with the dashed red line in case of the overshoot. Furthermore, a desirable value $f_{\mathrm{opt}_{i}}$ can be assigned to each performance measure representing the optimum that may be in between 0 and the maximum admissible value $f_{\Gamma, i}$. Formulating a minimization problem for a nominal plant with the parameters $\boldsymbol{\theta}_{0}$ leads to the following equation:

$$
J_{\boldsymbol{K}}^{\Gamma_{i}}=\left\{\begin{array}{ll}
\left|\frac{f_{i}\left(\theta_{0_{i}}, \boldsymbol{K}\right)-f_{\mathrm{opt}_{i}}}{f_{\Gamma_{i}}-f_{\mathrm{opt}_{i}}}\right| & f_{i}\left(\theta_{0_{i}}, \boldsymbol{K}\right)<f_{\Gamma_{i}} \\
1 & \text { else }
\end{array},\right.
$$

where $\Gamma_{i}$ corresponds to a particular performance index. Hence, the cost function value $J_{K}^{\Gamma_{i}}$ is the absolute distance between the measured performance $f_{i}\left(\theta_{0_{i}}, \boldsymbol{K}\right)$ and $f_{\text {opt }_{i}}$ that is evaluated over every sample of the grid mesh of $\boldsymbol{K}=\left\{K_{\mathrm{p}}, K_{\mathrm{i}}\right\}$. A normalization with the worst case distance $f_{\Gamma_{i}}-f_{\mathrm{opt}_{i}}$ restricts the performance value to the range of $[0 ; 1]$. Reaching the optimum value corresponds to a cost function value 0 and violating the maximum admissible value $f_{\Gamma_{i}}$ corresponds to the cost function value 1 . Due to the normalization all $m$ performance measures can be combined by a weighted sum:

$$
\begin{gathered}
J_{\boldsymbol{K}}^{\Gamma}=\left\{\begin{array}{ll}
\sum_{i}^{m} w_{i} J_{\boldsymbol{K}}^{\Gamma_{i}} & \forall J_{\boldsymbol{K}}^{\Gamma_{i}}<1 \\
1 & \text { else }
\end{array},\right. \\
\text { with } \omega_{i} \geq 0, \sum_{i}^{m} \omega_{i}=1 .
\end{gathered}
$$

The corresponding set $\boldsymbol{K}_{\Gamma}$, that meets the performance measures is:

$$
\boldsymbol{K}_{\Gamma}=\boldsymbol{K}\left(J_{\boldsymbol{K}}^{\Gamma}<1\right)
$$

\section{B. Robustness Measures}

Considering the plant parameters to be uncertain, that are bounded by upper and lower limits, leads to a hyper box $Q$ :

$$
Q=\left\{q_{i} \in\left[q_{i}^{-} ; q_{i}^{+}\right] \mid, i=\{1,2, \cdots, l\}\right\} .
$$

As stated in [12] the critical parameter sets can be found in the corners of the $Q$-box, as long it is an convex problem. The cost function is than extended to the following equation:

$$
J_{\boldsymbol{k}}^{\Gamma_{i}}= \begin{cases}\left|\frac{\max \left(f_{i}\left(Q_{\mathrm{E}}, k\right)-f_{\mathrm{opt}_{i}}\right)}{f_{\Gamma_{i}}-f_{\mathrm{opt}_{i}}}\right| & \forall f_{i}\left(Q_{\mathrm{E}}, \boldsymbol{k}\right)<f_{\Gamma_{i}} \\ 1 & \text { else }\end{cases}
$$
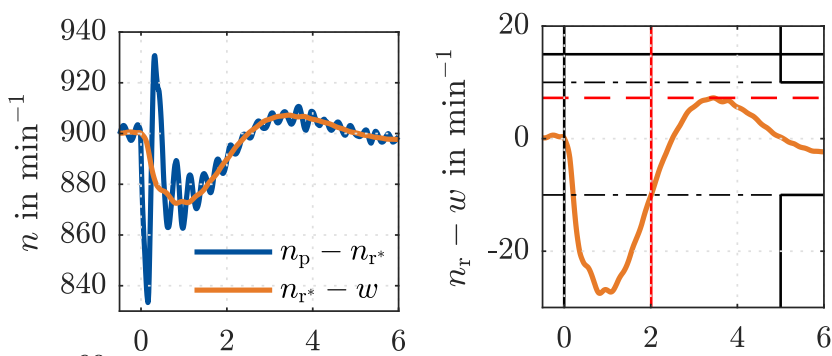

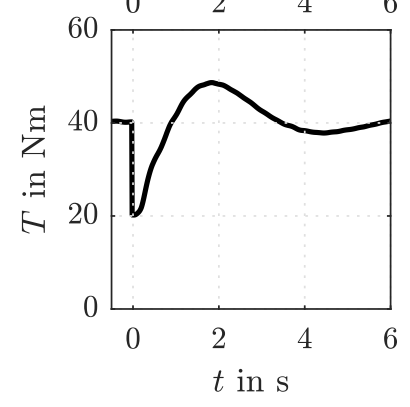

(a)

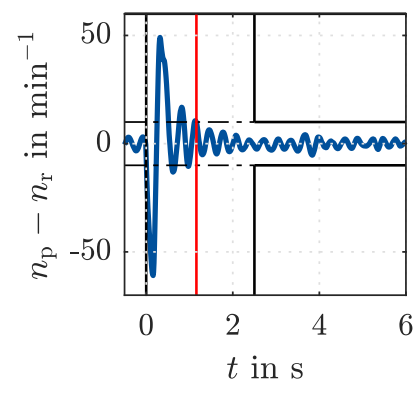

(b)
Fig. 2. (a) shows measured and processed engine speed $n_{\mathrm{p}}$, wheel speed $n_{\mathrm{r}}$ and the controller output $T_{\text {ecu }}$ of a negative disturbance step response. The signals that are shown in (b) are used in order to determine the performance measures. The signals are taken from a simulation with respect to the introduced model whose parameters have been identified and validated by means of real measurements of a passenger car.

A control parameter is robustly $\Gamma$-stable with respect to the required performance measure $\Gamma_{i}$ and the hyper box $Q$, if its cost function is smaller than 1 for all considered points of $Q_{\mathrm{E}}$. Expanding the $Q$-Box step-wise causes the $\Gamma$-stable area to shrink until there are no stabilizing parameters anymore. The area that remains at longest with increasing $Q$-box exhibits the greatest robustness and the area, that vanishes first consequently has the least robustness.

Keeping this idea in mind a robustness characterizing measure can be introduced. For this purpose the control parameters are regarded to be fixed at a particular value. Assuming the $Q$-Box is expanded by $n$ incremental steps, the interval of each uncertain parameter $q:\left[q^{-}, q^{+}\right]$is divided into equidistant subintervals of width $\Delta_{p}$ :

$$
p_{j}=j \Delta_{p}, \quad j=0, \ldots, n-1 .
$$

The $Q$-box is gradually increased with $n$ steps. For each step and parameter the following subinterval results:

$$
q_{i}^{ \pm}\left(p_{j}\right)=q_{i, 0}\left(1 \pm p_{i, j}\right) .
$$

Finally the level of robustness is defined as follows:

$$
R_{k}=\frac{z}{n},
$$

$z$ equals the number each time $J_{\boldsymbol{k}}^{\Gamma}\left(p_{j}\right)<1$. A parameter set with a robustness measure equal to 0 does not meet performance requirements for the nominal plant corresponding 
to $\boldsymbol{\theta}_{0}$. Regarding the other extreme a robustness measure equals $m$ fulfills the performance requirements as long as the parameters do not exceed the maximum assumed variation of $p=(n-1) \Delta_{p}$.

\section{Design Approach}

Within the scope of the controller design methodology that is pictured in Fig. 3 three aspects are considered and need to be defined by the user:

- Physical uncertainties of the plant, which are represented by the hyper box $Q$. It contains $l$ parameters, that are assigned to a nominal value $q_{i, 0}$ within an interval $\left[q_{i}^{-} ; q_{i}^{+}\right]$. Initially, only the nominal values are regarded.

- Control parameters $K$, that need to be designed. In this application a PI-controller with the control parameters $k_{\mathrm{p}}$ and $k_{\mathrm{i}}$ are designed. The spanned parameter space within the intervals $\left[k_{j}^{-} ; k_{j}^{+}\right]$is divided by $25 \times 25$ grid points.

- Performance requirements $\Gamma_{i}$ containing the settling time and overshoot with respect to $n_{1}$ and $n_{2}$ in Fig 2.

In the design step the nominal plant is simulated and evaluated with respect to the performance measures for each sample within the set of $\boldsymbol{K}$. On this basis a performance map can be obtained. An accurate resolution of the maps requires a high simulation effort, that can be minimized by means of the parameter space approach (PSA). The overall aim of the PSA is to have a rough estimation of the $\Gamma$-stabilized region using a reduced linear model. For this purpose It provides $\Gamma$-stable boundaries, that are mapped from $s$-domain into the parameter space. They can be seen as preliminary conditions, that must be full-filled. Otherwise the simulation for certain parameter samples will be skipped and the corresponding performance values are saved as NaN. The applied system on the PSA is based on an integrator plant. This is admissible in the sense as the system is ruled by the low frequency behaviour, which is sufficiently described by this simplified model. Hence, the parameter space can reduced a lot.

Considering uncertain parameters each control parameter sample needs to be simulated with the worst case combination, that arises from the given $Q$-box. In a convex problem formulation it can be found in the corners of the intervals. Instead if gridding the entire $Q$-box only $2^{l}$ points are representatively checked. However, the number of corner points rises exponentially with $l$. Hence, it is likewise reasonable to reduce the number of uncertain parameters that can be achieved with the aid of a sensitivity analysis (SA). Due to the large intervals of the $Q$-box and because of nonlinear parameter dependencies a global approach must be applied. In this paper a variance based sensitivity analysis is performed [13]. This approach provides in addition unified measures in the range between 0 and 1 , representing a negligible respectively a significant influence of the uncertain parameters on the performance measures. Finding a proper

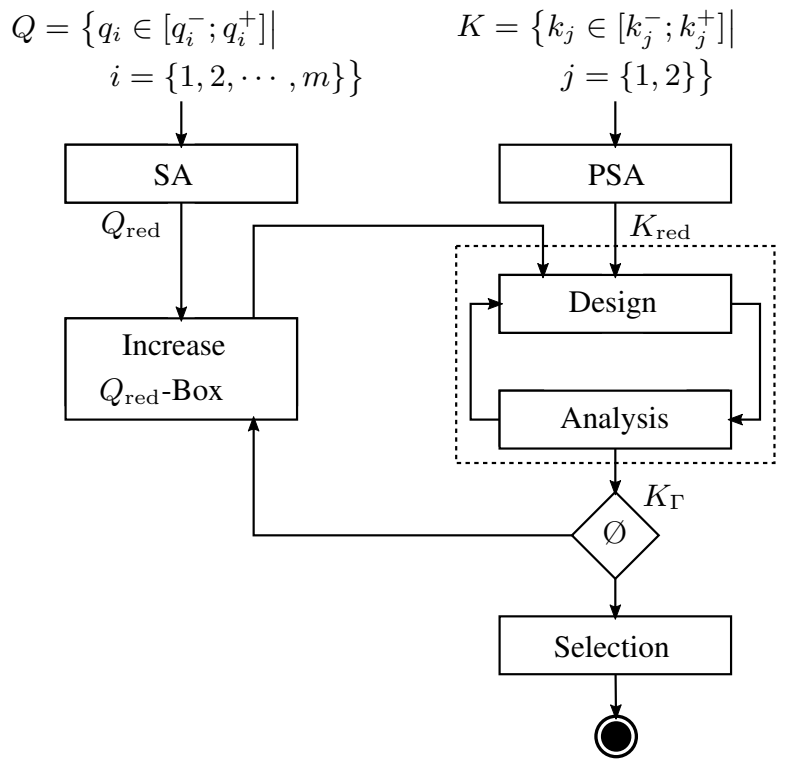

Fig. 3. Design Approach.

threshold that separates significant parameters from negligible parameters is not easy to suggest. In order to analyse the proposed design approach for this application, we will begin with a set of two uncertain parameters and subsequently increase the number of uncertain parameters.

It must be noticed that in case of a non-convex formulation, the worst case parameter combination may occur along the egdes of the $Q$-box. Because of this reason subsequently to the design a analysis step must be performed. A selected sample within the preliminary set of $\boldsymbol{K}_{\Gamma}$ is fixed in order to test a randomly chosen set on the edges of $Q$. If any samples of the test set fail, they have to pass the design process additionally. Otherwise the approach will be proceed with the resulting set $\boldsymbol{K}_{\Gamma}$. Assuming that after the design and analysis step $K_{\Gamma} \neq \varnothing$ is obtained, the $Q$-box will be extended by increasing the intervals about $\Delta_{p}$ with respect to (14). This is proceed until $\boldsymbol{K}_{\Gamma}=\varnothing$ is satisfied. In every further design and analysis iteration over the expanding $Q$ box the previously determined set of $K_{\Gamma}$ is taken.

\section{RESUlTS}

In this section the results of the SA, the PSA and the controller design are given. The applied methods, the SA and PSA, are also briefly introduced.

\section{A. Sensitity Analysis}

By means of the SA the influence of the variation of particular inputs $\boldsymbol{X}=\left\{X_{1}, X_{2}, \ldots, X_{k}\right\}$ shall be quantified with respect to particular outputs $Y$. Throughout this paper the inputs are physical plant parameters and the outputs are performance measures at a fixed value of $K_{\mathrm{p}}$ and $K_{\mathrm{i}}$. Each input is described by an individual and unified distribution 
$\mathcal{U}(a, b)$. Furthermore, nonlinear dependencies lead to mutual interactions, that need to be revealed. These challenges are met by using a variance-based SA. With respect to the sensitivity values two kinds of measures need to be distinguished [13]:

- main effect $S_{\mathrm{H}}$ : describes the influence that can be assigned to a single input when varying only one parameter at a time,

- total effect $S_{\mathrm{T}}$ : describes the influence that is caused besides the main effect by any interactions of this input with other inputs.

The fundamental calculation of the sensitivity indices are:

$$
\begin{aligned}
S_{\mathrm{H}_{i}} & =\frac{V\left(E\left(Y \mid X_{i}\right)\right)}{V(Y)} \\
S_{\mathrm{T}_{i}} & =1-\frac{V\left(E\left(Y \mid \boldsymbol{X}_{-i}\right)\right)}{V(Y)}
\end{aligned}
$$

In this formula the numerator is defined as a conditional variance with respect to the input $P_{i}$ that is divided by the absolute variance of the output $Y$. The expected mean is obtained when one input, $X_{i}$, is fixed to a particular value $x_{i}^{*}$ and all other inputs are varied such as $E_{\boldsymbol{X}_{-i}}\left(Y \mid X_{i}\right)$ and in short notation $E\left(Y \mid X_{i}\right)$. Finally the variance is taken over all possible values of $X_{i}$ [13]. Further important properties are:

$$
\begin{aligned}
S_{\mathrm{H}_{i}} & \in[0 ; 1], \\
\sum_{i=1}^{k} S_{\mathrm{H}_{i}} & =1, \\
S_{\mathrm{T}_{i}} & \approx S_{\mathrm{H}_{i}} \quad \text { in absence of interactions } \\
S_{\mathrm{T}_{i}} & >>S_{\mathrm{H}_{i}} \quad \text { in presence of interactions. }
\end{aligned}
$$

In this paper the sobol approximation is used that is based on a monte-carlo simulation. According to the amount of inputs $k$ and an appropriate number of independent samples $n$ the following number of simulation runs are required:

$$
N=n(k+2) \text {. }
$$

The parameters are uniformly distributed with different levels of uncertainty:

$$
\mathcal{U}\left(q^{-} ; q^{+}\right), \quad q^{ \pm}=q_{0}(1 \pm p)
$$

The gain of the engine $K_{\text {eng }}$ and the vehicle mass $J_{\mathrm{r}}$ are assumed to vary about $p_{1}=20 \%$, which may be caused by loading of the car respectively a improper engine calibration at a certain operation point. The remaining parameters $J_{\mathrm{p}}, J_{\mathrm{s}}, c_{\mathrm{clu}}, \varepsilon_{\mathrm{clu}}, d_{\mathrm{clu}}, T_{\mathrm{clmb}}, c_{\mathrm{drv}}, \varepsilon_{\mathrm{drv}}, d_{\mathrm{drv}}$ are assumed to have relatively low variation of $p_{2}=5 \%$, which may occur only by manufacturing tolerances and ageing. As the information about real parameter variations are not available, these are just assumptions that were made by the authors of this paper in order to exemplary show the methodology. According to $n=2000$ samples the results of the SA are shown

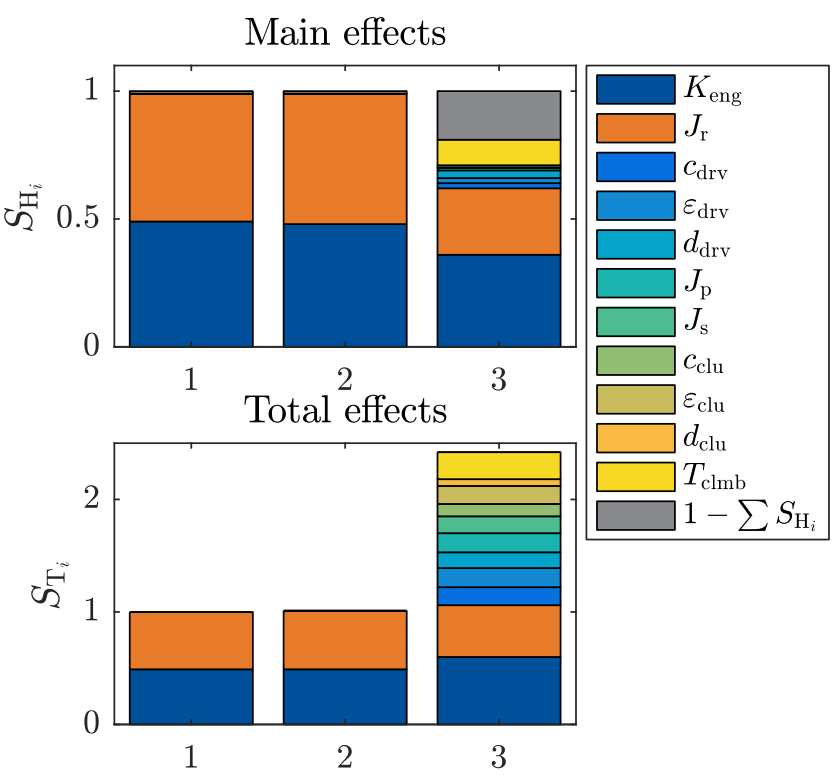

Fig. 4. Main effects and total effects of the SA considering uncertainty intervals that correspond to $p_{1}$ and $p_{2}$. The indices on the $x$-axis correspond to the performance measures.

in Fig. 4 in bar charts. Regarding the first two performance measures, there is no interactions between the parameters, which is indicated by $\sum_{i=1}^{k=11} S_{i}^{\Gamma_{1}}=1$ and $\sum_{i=1}^{k=11} S_{i}^{\Gamma_{2}}=1$. In both cases $K_{\text {eng }}$ and $J_{\mathrm{r}}$ show a significant influence and the other parameters are negligible. However the case is different concerning the third performance index which is indicated by $\sum_{i=1}^{k=11} S_{i}^{\Gamma_{3}}=0.75$. Regarding the main effects besides $K_{\text {eng }}$ and $J_{\mathrm{r}}$ an influence of $T_{\text {clmb }}$ must be noticed that is about 0.09 . Regarding the total effect $d_{\mathrm{drv}}$ has the smallest value of 0.06 . The other parameters show values in the range of $0.14<S_{\mathrm{T}}-S_{\mathrm{H}}<0.18$. As mentioned in section III-C we start with the set of $K_{\text {eng }}$ and $J_{\mathrm{r}}$ and extend it subsequently from top down accordingly to the values of $S_{\mathrm{T}_{i}}^{\Gamma_{3}}$. The results are discussed in section IV-C.

\section{B. Parameter Space Approach}

In order to reduce the number of grid points boundary lines in parameter space are required that cover some performance requirements. For this reason the parameter space approach is applied in this paper. This approach provides an analytical way to find these boundaries. Two aspects need to be considered. First is that the PSA maps so-called $\Gamma$-stabilized regions from $s$-domain into parameter space. However, it is possible to find an equivalent mapping between performance requirements in $s$-domain and physically interpretable requirements in time domain. The second aspect is that an analytical solution can only be found for simple models. With respect to the results of [14] accurate results can be found for the step response of the reference variable considering an integrator plant. Based on that approach the following mapping rules 


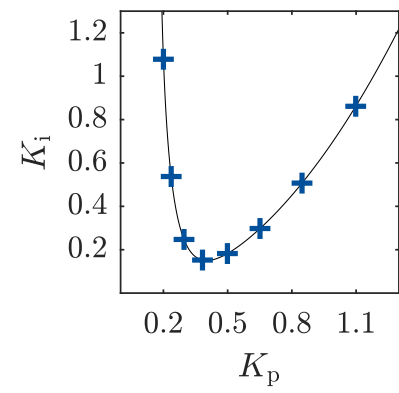

(a) Parameter space

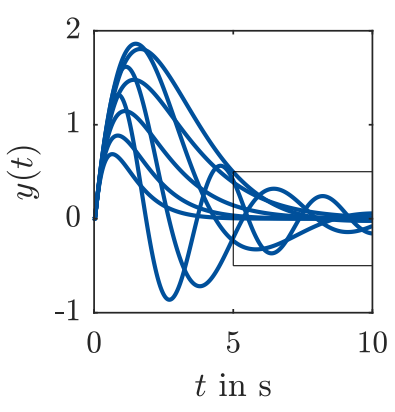

(b) Disturbane step response
Fig. 5. Applying the PSA on a simplified model considering (25) and (26) with $\varepsilon=0.5$ and $t_{\varepsilon}=5 \mathrm{sec}$.

in $s$-domain can be established for the disturbance step:

$$
\sigma=\frac{\ln (\varepsilon K \omega)}{t_{\varepsilon}},
$$

It must be noticed that the underdamped case $\zeta \in[0 ; 1]$ is considered with respect to the following reduced model:

$$
\begin{aligned}
P(s) & =\frac{K}{s} e^{-s T_{\mathrm{D}}}, \quad C(s)=\frac{K_{\mathrm{p}} s+K_{\mathrm{i}}}{s} \\
\Rightarrow G(s) & =\frac{P(s)}{1+P(s) C(s)},
\end{aligned}
$$

with $K=\frac{30}{\pi J}$ and $J=J_{p}+J_{s}+J_{r} / i_{g}^{2}$. The resulting boundary in parameter space with respect to $t_{\varepsilon}=5 \mathrm{sec}$ and $\varepsilon=0.5$ is shown in Fig.5. By means of the PSA the total amount simulation runs can be reduced from 625 to 327 . Notice the conservatism for $K_{\mathrm{p}}>0.5$ due to the fact that in (24) the underdamped case is considered.

\section{Design and Analysis}

The presented approach is applied on a test case, that is shown in Fig. 2. The idle speed controller is enabled and drives the vehicle with constant idle speed. After a negative step of a disturbance torque applied on the engine side, the closed loop behavior is evaluated by means of three introduced performance measures. Fig. 6 illustrates the control parameter space, which is obtained by $25 \times 25$ equidistant values within the shown grid mesh. The performance is divided into six performance levels. According to the color bar, the best performance region is black colored and the worst performance region is white colored. In Fig. 6(d), the combined total performance is shown. For the nominal case the optimal parameterization is obtained with $K_{\mathrm{p}}=0.4$ and $K_{\mathrm{i}}=0.5$. It can be stated that the overshoot of $n_{1}$ and the settling of $n_{2}$ can be adjusted mainly over $K_{\mathrm{p}}$, while the settling time of $n_{1}$ reacts more sensitive to changes of $\boldsymbol{K}_{\mathrm{i}}$. In total, there is a quit spacious $\Gamma$-stabilizing region in parameter space considering the nominal plant. Increasing the Q-Box the region of $\boldsymbol{K}_{\Gamma}$ shrinks, as shown in Fig 3. Considering only two uncertain parameters the case of $K_{\Gamma}=\varnothing$ is

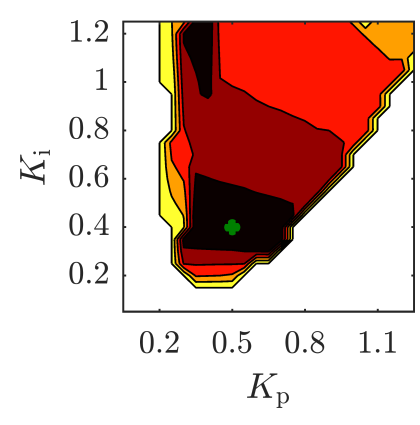

(a)

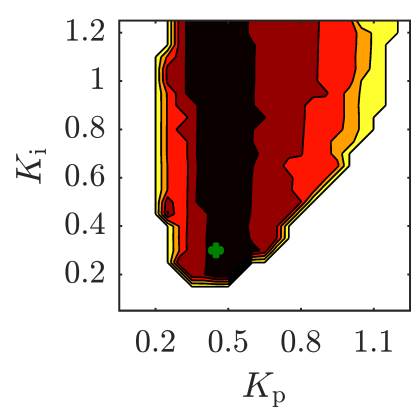

(c)

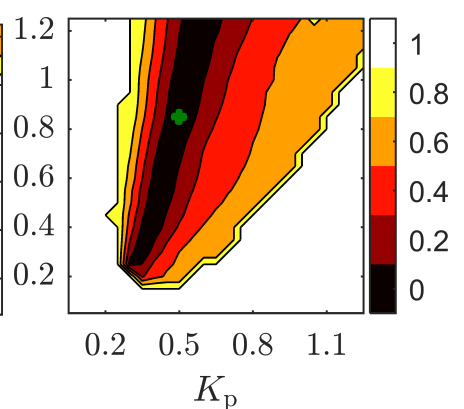

(b)

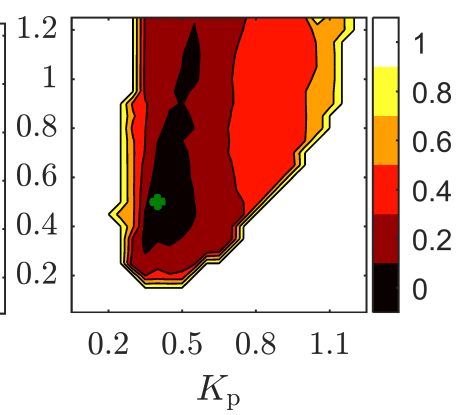

(d)
Fig. 6. Nominal performance portraits. (a) and (b) correspond to the settling time and overshot of $n_{1}$, (c) corresponds to the settling time of $n_{2}$ and (d) shows the overall performance portrait.

reached at $p=70 \%$. The robust parameterization is obtained inside a small band with $K_{\mathrm{p}}=0.35$ and $K_{\mathrm{i}}=[0.85 ; 1]$. In comparison with seven uncertain parameters almost the same robust parameterization is determined with $K_{\mathrm{p}}=0.4$ and $K_{\mathrm{i}}=0.9$. However it must be noticed that $K_{\Gamma}=\varnothing$ appears already at $p_{1}=60 \%$ respectively $p_{2}=15 \%$. Due to the low sensitivities of the neglected uncertain parameters a significantly different result is not conceivable.

\section{Parameter Secelection and Discussion}

By reaching $\boldsymbol{K}_{\Gamma}=\varnothing$ the algorithm is finished and some data is available to select a particular control parameterization. The following strategies can be derived:

1) optimal performance $K_{\mathrm{opt}}=[0.4 ; 0,5]$ : considering the nominal system with $j=0$ the parameter sample with the lowest value of $J_{K}^{\Gamma}$ is selected.

2) robust performance $K_{\text {rob }}=[0.4 ; 0,9]$ : considering the results from $j=n-1$ the parameter sample that still remains in $\boldsymbol{K}_{\Gamma}$ is selected. If $\boldsymbol{K}_{\Gamma}$ contains more than one sample the $Q$-box has to be expanded in smaller steps of. It is also conceivable to select a parameter with the biggest distance to the boundaries of $\boldsymbol{K}_{\Gamma}$.

3) optimal performance with certain robustness level $K_{\text {hyb }}=[0.4 ; 0,7]$ and $w=0.5$ : with respect to the selection from (1) and (2) a hybrid solution can be obtained. According to a weighting $w$ a linear interpolation between these two samples is determined. 


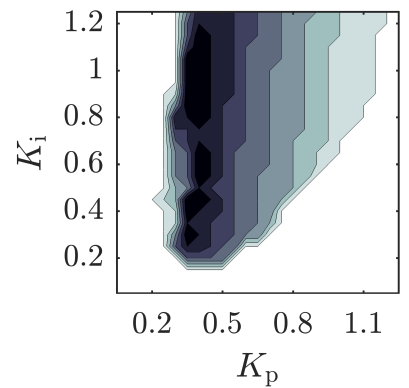

(a)

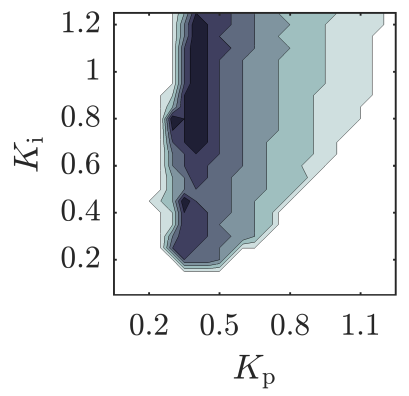

(c)

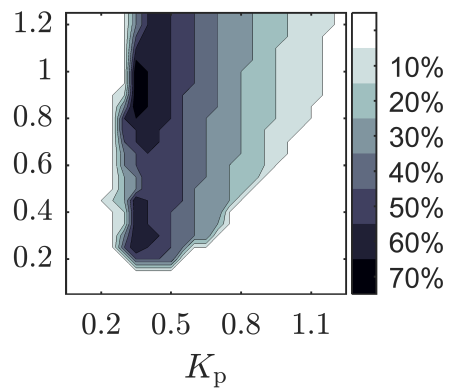

(b)

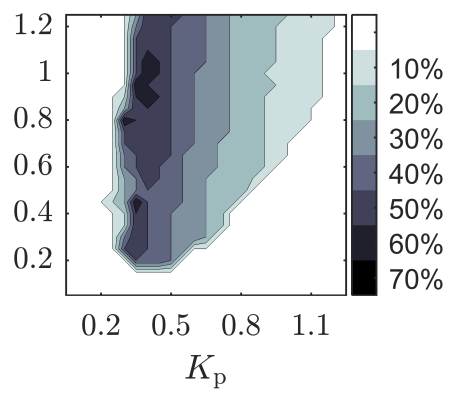

(d)
Fig. 7. Robust performance portait considering two uncertain parameters and subsequently added further uncertain parameter. The following sets correspond to the graphs (a)-(d): $\left\{K_{\mathrm{eng}}, J_{\mathrm{r}}\right\}$, $\left\{K_{\text {eng }}, J_{\mathrm{r}}, T_{\text {clmb }}\right\}, \quad\left\{K_{\text {eng }}, J_{\mathrm{r}}, T_{\text {clmb }}, J_{\mathrm{p}}, c_{\mathrm{drv}}, \varepsilon_{\mathrm{clu}}\right\} \quad$ and $\left\{K_{\mathrm{eng}}, J_{\mathrm{r}}, T_{\mathrm{clmb}}, J_{\mathrm{p}}, c_{\mathrm{drv}}, \varepsilon_{\mathrm{clu}}, J_{\mathrm{s}}\right\}$. The colorbar indicates the admissible regions of the control parameters corresponding to the uncertainty levels of $p_{1}$ and $p_{2}=p_{1} / 4$.

Comparing the results, the difference between three solution $K_{\text {opt }}, K_{\text {rob }}, K_{\text {hyb }}$ seems to be very small. Regarding the performance portrait in Fig. 6(d) the parameterization with $K=[0.6 ; 0.4]$ has an insignificantly performance such that level of $J=0$ is still given. Comparing this solution again with the performance portrait in Fig. 7(d) a considerable smaller robustness value of $30 \%$ instead of $60 \%$ is achieved. In the opposite case comparing $K_{\text {rob }}=[0.4 ; 0,9]$ with the performance portrait a performance value of 0.2 remains. In summary the elaborated methodology provides deep insights and a valid suggestion for a controller application. On this basis a trade-off between performance and robustness can be obtained.

\section{CONCLUSION}

In conclusion, a model-based controller design approach is presented in this work that is applicable on any controller structure. The user is able to define performance measures in order to find an optimal parameterization. Furthermore by introducing uncertain intervals that are assigned to plant parameters robust control parameters can be obtained. In the sense of robust performance it is necessary to define thresholds according to the performance measures in order to accept of neglect certain parameter samples. Under this condition typically used criteria like the integral absolute error (IAE), total variance (TV) are also considerable. However due to the grid search it must be noticed that this approach means a high computational effort that scales exponentially with the number of uncertain parameters. As a countermeasure on one hand the PSA is integrated in order to restrict the grid points in parameters space. On the other hand a global sensitivity analysis is performed in order to reduce the dimensions of the $Q$-box. With respect to the results of the SA the main and total effects can be quantitatively compared with each other, but finding a viable threshold in order to neglect uncertain parameters is a matter of required accuracy and computing resources. By means of the resulting top down sequence that corresponds to the SA a systematical procedure is shown. Furthermore in terms of time consuming vehicle tests in real applications the results offer insights about the system and can be used for optimizing a design of experiment.

\section{REFERENCES}

[1] L. Xiao and F. Gao, "A comprehensive review of the development of adaptive cruise control systems," Vehicle System Dynamics, vol. 48, no. 10 , pp. $1167-1192$, Oct. 2010.

[2] A. Walter, M. Murt, U. Kiencke, S. Jones, and T. Winkler, "Compensation of sub-harmonic vibrations during engine idle by variable fuel injection control," IFAC Proceedings Volumes, vol. 41, no. 2, pp. 9459-9466, 2008.

[3] M. R. Gharib and M. Moavenian, "A New Generalized Controller for Engine in Idle Speed Condition,” p. 9, 2012.

[4] G. J. Naus, M. A. Beenakkers, R. G. Huisman, M. J. van de Molengraft, and M. Steinbuch, "Robust control of a clutch system to prevent judderinduced driveline oscillations," Vehicle System Dynamics, vol. 48, no. 11, pp. 1379-1394, Nov. 2010.

[5] H. Zhang, Y. Zhang, and C. Yin, "Hardware-in-the-Loop Simulation of Robust Mode Transition Control for a SeriesParallel Hybrid Electric Vehicle," IEEE Transactions on Vehicular Technology, vol. 65, no. 3, pp. 1059-1069, Mar. 2016.

[6] T. Hrycej, Robuste Regelung. Berlin, Heidelberg: Springer Berlin Heidelberg, 2018.

[7] M. Huba, "Performance measures, performance limits and optimal PI control for the IPDT plant," Journal of Process Control, vol. 23, no. 4, pp. 500-515, Apr. 2013.

[8] F. Schütte, Automatisierte Reglerinbetriebnahme für elektrische Antriebe mit schwingungsfähiger Mechanik, ser. Berichte aus der Steuerungs- und Regelungstechnik. Shaker, 2003.

[9] S. Zemke, Analyse und modellbasierte Regelung von Ruckelschwingungen im Antriebsstrang von Kraftfahrzeugen. VDI-Verlag, 2012.

[10] M. Nordin, J. Galic', and P.-O. Gutman, "New models for backlash and gear play," International Journal of Adaptive Control and Signal Processing, vol. 11, no. 1, pp. 49-63, 1998.

[11] E. Popp, M. Tantau, M. Wielitzka, T. Ortmaier, and D. Giebert, "Frequency Domain Identification and Identifiability Analysis of a Nonlinear Vehicle Drivetrain Model," in 2019 18th European Control Conference (ECC). Naples, Italy: IEEE, June 2019, pp. 237-242.

[12] J. Ackermann, Robust Control: the Parameter Space Approach. Springer London, 2002.

[13] A. Saltelli, S. Tarantola, F. Campolongo, and M. Ratto, Sensitivity Analysis in Practice. Chichester, UK: John Wiley \& Sons, Ltd, Feb. 2002. [Online]. Available: http://doi.wiley.com/10.1002/0470870958

[14] E. Popp, M. Wielitzka, D. Giebert, and T. Ortmaier, "Design of a PI-Controller Based on Time-Domain Specification Utilizing the Parameter Space Approach," in 2020 21st IFAC World Congress, in press. 